\title{
Wavelet Based and Statistical EEG Analysis in Patients with Schizophrenia
}

\author{
Musa Yilmaz \\ Electrical and Electronics Engineering, Batman University, Bati Raman Campus, Batman 72070, Turkey
}

Corresponding Author Email: musa.yilmaz@batman.edu.tr

https://doi.org/10.18280/ts.380523

Received: 16 March 2021

Accepted: 22 September 2021

\section{Keywords:}

EEG data, schizophrenia, wavelet analysis, statistics

\begin{abstract}
Schizophrenia, which is considered a serious mental disorder and a psychological illness, is quite common in society today. Schizophrenia manifests itself with disordered thought development, hallucinations, and different behaviours and reactions. In this study, EEG data were collected and analyzed on a total of 84 subjects diagnosed with normal and schizophrenia. EEG data used as a diagnostic tool with low-resolution level has been used to distinguish between schizophrenic and normal individuals. First of all, statistical methods were used in the analyzes, and also frequency properties of the data were extracted by Wavelet analysis. As a result of the analysis, statistical findings include characteristics that distinguish between diagnosed schizophrenia and normal individuals. In addition, the findings obtained as a result of the Wavelet analysis were determined to distinguish between normal and schizophrenic individuals. While the mean used in statistical analysis takes the value 1.6 for normal individuals, it takes the value 2.9 for individuals diagnosed with schizophrenia. Also, in the results of Continuous Wavelet (CW) Analysis, very important findings were obtained in terms of detection in scale 16 and 64 bands.
\end{abstract}

\section{INTRODUCTION}

Schizophrenia, thought to be caused by environmental and genetic factors, is in the group of common psychological diseases [1-3]. People with schizophrenia have difficulty in evaluating reality, controlling their emotions, and communicating with their environment. The most obvious symptoms are suspicion and hallucinations. It is defined as a chronic disease and tends to worsen gradually if left untreated $[4,5]$.

Schizophrenia symptoms in adolescents are first discovered in schools. The most important symptoms of the patients are low educational performance at school, lack of motivation, irritable behaviour, depressed mood, sleep disorder, and a living environment away from their environment and family as an asocial personality [6]. Patients with schizophrenia can often survive without realizing that they are ill [7]. It has been established that most schizophrenic patients are suicidal. Although the exact cause of schizophrenia is not known, it is thought to be factors such as genetic factors, complications during childbirth, malnutrition or toxic chemical drug intake [8]. Schizophrenia is a treatable disease [9]. In the first stage of treatment, drug therapy is used, and the psychiatrist checks whether the patient responds to the treatment within the framework of a long-term treatment plan. With continuous psychiatrist control, the effect of the dose of antipsychoticbased drugs on the treatment process can be examined, and the treatment of the patient can be provided [10]. The important thing here is to identify the patient with schizophrenia. Because many schizophrenic patients can continue their lives without knowing that they are sick. For this purpose, diagnoses using EEG provide great convenience [11]. There are many studies in the literature in which EEG signs are used to detect psychological disorders. The most common studies in this area have been done by applying data on patients with depression, epilepsy, and schizophrenia [12]. While some of the studies conducted are sign-based studies, most of them are aimed at distinguishing signs using artificial intelligence methods [13, 14].

In this study, a diagnosis of schizophrenia was made, which compared and analyzes by evaluating the sign-based Wavelet analysis and statistical data of normal control individuals.

\section{MATERIALS AND METHODS}

The data collected in this study (http://brain.bio.msu.ru/eeg schizophrenia.htm) were obtained from the data bank. In the study, EEG data were collected from adolescents aged 10-14 years. During the data collection phase, data from 84 adolescent children were collected according to clinical results. The data collected from these 84 people included 39 healthy individuals and 45 individuals diagnosed with Schizophrenia. Data were collected from 84 persons with their eyes closed [15]. No drug was given to the individuals whose data was collected during the data collection, and the data were recorded using a total of 6 electrodes. The sampling frequency of the EEG device is 128 $\mathrm{Hz}$, the electrode impedance is $10 \mathrm{kOhm}$ and the measurement time is 1 minute. The collected data are 7680 and shared publicly in the data bank (http://brain.bio.msu.ru/eeg_schizophrenia.htm.), and also analyzed. Electrode distribution is given in Figure 1 [16]. In Figure 2, the time-amplitude graph of the data collected from the F3 electrode from the normal subject is given.

In Figure 3, the time-amplitude graph of the data collected from the subject diagnosed with schizophrenia from the F3 electrode is given. 


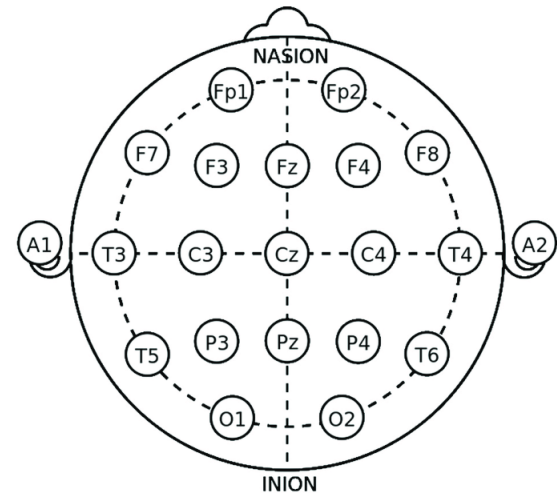

Figure 1. Placement of the EEG electrodes location and 1020 international system [16]

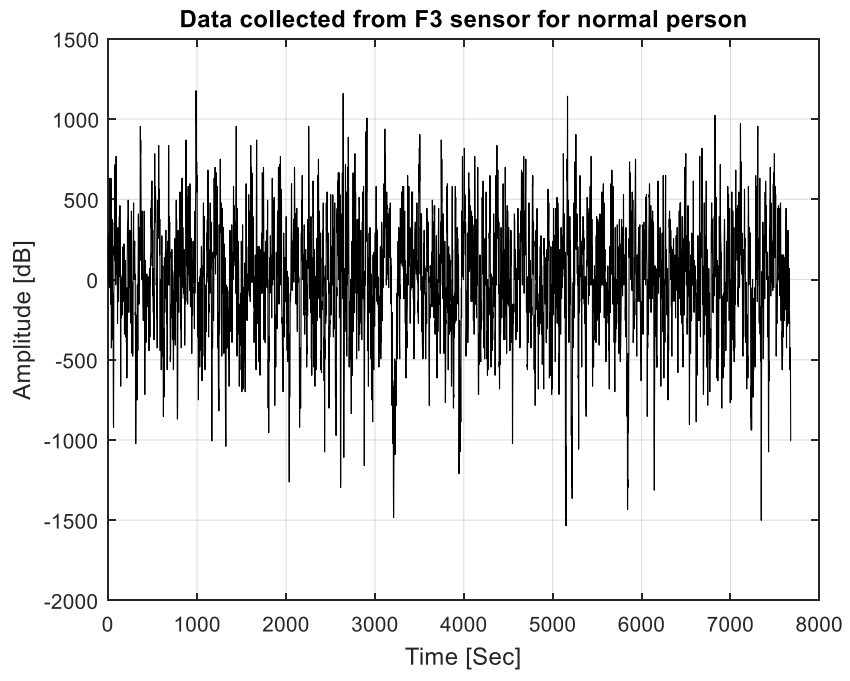

Figure 2. EEG data from F3 sensor (for normal participant)

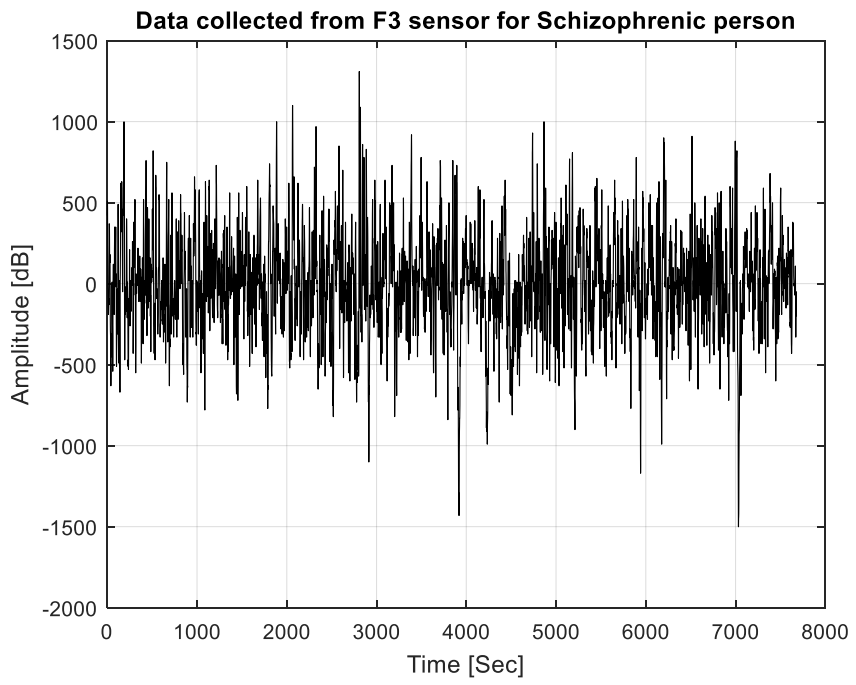

Figure 3. EEG data from F3 sensor (for schizophrenic person)

\section{MATHEMATICAL BACKGROUND}

In this section, basic statistical equations for Wavelet Analysis and Statistical parameters are explained. In the next section, the applications of these mathematical methods have been made and compared.

\subsection{Wavelet analysis}

Computers with high processing power in Fourier analysis used in signal processing analysis and the development of programming techniques have shown great improvement [17]. Today, Wavelet analysis finds use in many areas [18, 19]. With this analysis, time, amplitude and details can be analyzed, and low and very high-frequency regions can be easily detected [20, 21]. Wavelet analysis is the most preferred method among time-frequency analysis methods to reveal regional features in signals [22]. This method is quite successful in the analysis of Non-linear signals compared to the Short Time Fourier Transform. Wavelet analysis is divided into two basic parts as the discrete and continuous wavelet transform [23]. In discrete wavelet transform, the signals are divided into sub-bands, while in continuous wavelet transform, the frequency bands of the data can be examined in terms of time, frequency and scale by using the displacement parameters of the data [24]. In this study, analyzes were made using the CWT method. CWT is given in Eq. (1).

$$
W(a, b)=\frac{1}{\sqrt{a}} \int_{-\infty}^{+\infty} f(x) \varphi\left(\frac{x-b}{a}\right) d x
$$

where, a is the scale parameter, $b$ is the transformation parameter and is the main wavelet.

$$
W(m, n)=2^{-\left(\frac{m}{2}\right)} \sum_{t=0}^{T-1} f(t) \varphi\left(\frac{t-n 2^{m}}{2^{m}}\right)
$$

In wavelet analysis T signal length, transformation and scale parameters as a function of integer values, a and be values are given in Eq. (1) and Eq. (2) [25, 26].

\subsection{Mean}

It is the most basic function used to see how data is distributed in statistical analysis. It is used to detect the central points of the data set. The mean is shown in Eq. (3) $[27,28]$.

$$
\mu=\frac{1}{N} \sum_{i=1}^{n} x_{i}
$$

\subsection{Standard deviation}

Standard deviation is defined as the measure of how the data propagates from the mean, that is, the average distance between each quantity and the mean. The low standard deviation data set tends to close to its mean, while the high standard deviation tends to take a wider range of values from the data points. The standard distribution equation is given in Eq. (4) below [27, 28].

$$
\sigma=\sqrt{\frac{1}{N} \sum_{i=1}^{n}\left(x_{i}-\mu\right)^{2}}
$$

\subsection{Variance}

Variance is the square of the mean distance between each quantity and the mean. So it is the square of the standard deviation. Variance, which is also defined as a measure of how 
a data set is distributed, is given in Equation 5. Here, S2 sample variance, $x i$ is the value of the one observation, $\dot{x}$ the mean value of all observations, and $\mathrm{n}$ is the number of observations $[27,28]$.

$$
S^{2}=\frac{\sum\left(x_{i}-\bar{x}\right)^{2}}{n-1}
$$

\subsection{Skewness}

Skewness gives information about whether a distribution is normal or not. It is done to test the distribution of the score in a certain data group in the form of a normal or bell curve. Skewness measures the symmetry of distribution, whereas Kurtosis indicates the abundance of samples at the midpoint of the distribution. The Skewness expression is given in Eq. (6) $[27,28]$.

$$
c=\frac{\sum_{i=1}^{N}(x(i)-\mu)^{3}}{N \sigma^{3}}
$$

\subsection{Kurtosis}

Outliers on data can be detected by skewness and kurtosis values. Since skewness includes the third moment of the distribution, kurtosis includes the fourth moment. In a symmetrical distribution, kurtosis is evident in the tail parts of the graph. Kurtosis and skewness do not have a unit, they are expressed by Eq. (7) $[27,28]$.

$$
k=\frac{\sum_{i=1}^{N}(x(i)-\mu)^{4}}{N \sigma^{4}}
$$

\section{APPLICATION ON THE DATA}

The results of statistical analysis of individuals with normal (control) and schizophrenia are given in Table 1 below. As examined in Table 1, all statistical values of individuals diagnosed with control and schizophrenia give quite decisive results.

The histogram graphic of the EEG signals belonging to the participants determined as the control group is given in Figure 4. In Figure 5, a histogram graph of EEG data of individuals with schizophrenia is given. When both graphs are examined, it is seen that both groups show a nominal distribution, but the normal group has a lower amplitude than the schizophrenic group.

The Pseudo-Spectrum Analysis graph is given in Figure 6 for normal participants who do not have schizophrenia. It is seen here that there are two short oscillations between 90-130 $\mathrm{dB}$ (normalized between 0 and $0.4 \mathrm{~Hz}$ ). In Figure 7, it can be seen that these oscillations increase between the same amplitude and frequency values. Similarly, while it takes a value of $45 \mathrm{~dB}$ for normal participants in the fading of the graph, this value decreases to $35 \mathrm{~dB}$ in participants with schizophrenia. This situation is similar to the values taken from other electrodes.

Figure 8 shows the Probability analysis chart for Normal Distribution without schizophrenia patients. The same analysis is given for the individual diagnosed with schizophrenia in Figure 9. When both graphs are examined, the probability function graph for the control group is on the axis curve, while it deviates from the axis to the right in the range of 1000-1500 data in schizophrenic individuals. This is a very distinctive chart for analysis done in a normal distribution. Such deviations are similar for data from other electrodes.

In Figure 10 and Figure 11, power spectrum analysis was performed first for the control group and then for individuals diagnosed with schizophrenia, respectively. The results of this analysis differ for individuals diagnosed with control and schizophrenia. Especially oscillations in the range of 100-101 contain features that distinguish between patient and control groups.

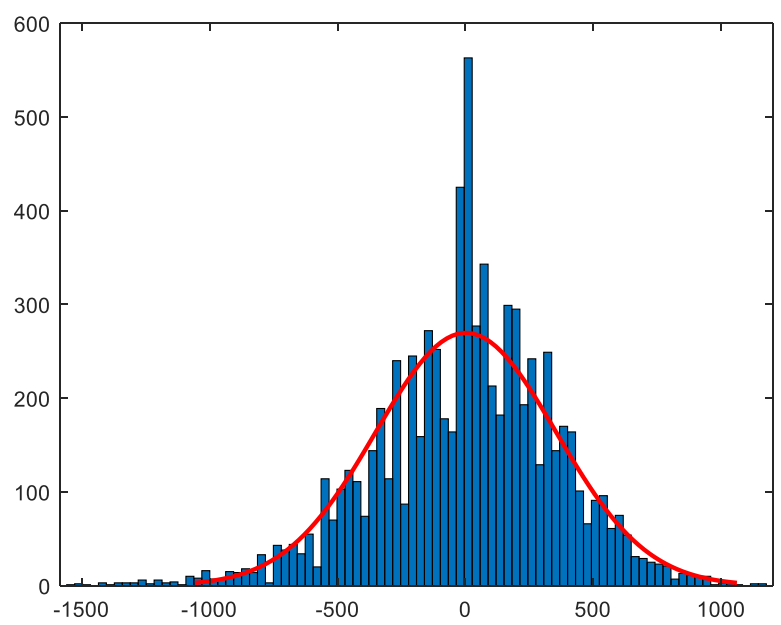

Figure 4. Histogram distribution for normal participants

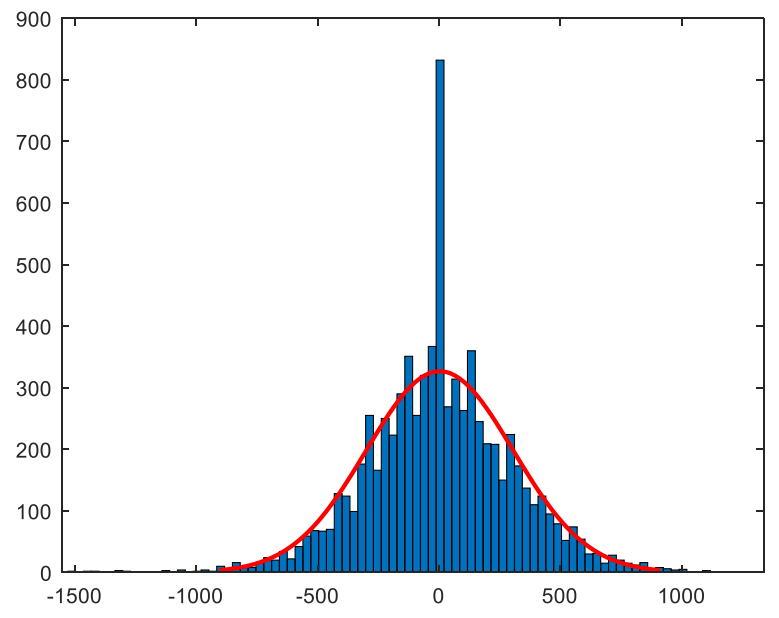

Figure 5. Histogram distribution of schizophrenic patients data

Table 1. Statistical analysis of individuals with control and schizophrenia

\begin{tabular}{cccccc}
\hline & Mean & Standard & Skewness & Kurtosis & Variance \\
\hline Normal People & 1.6077 & 353.3350 & -0.3800 & 3.7347 & $1.2485 \mathrm{e}+05$ \\
Şizofrenik People & 2.9961 & 302.8922 & -0.1023 & 4.2657 & $9.1744 \mathrm{e}+04$ \\
\hline
\end{tabular}




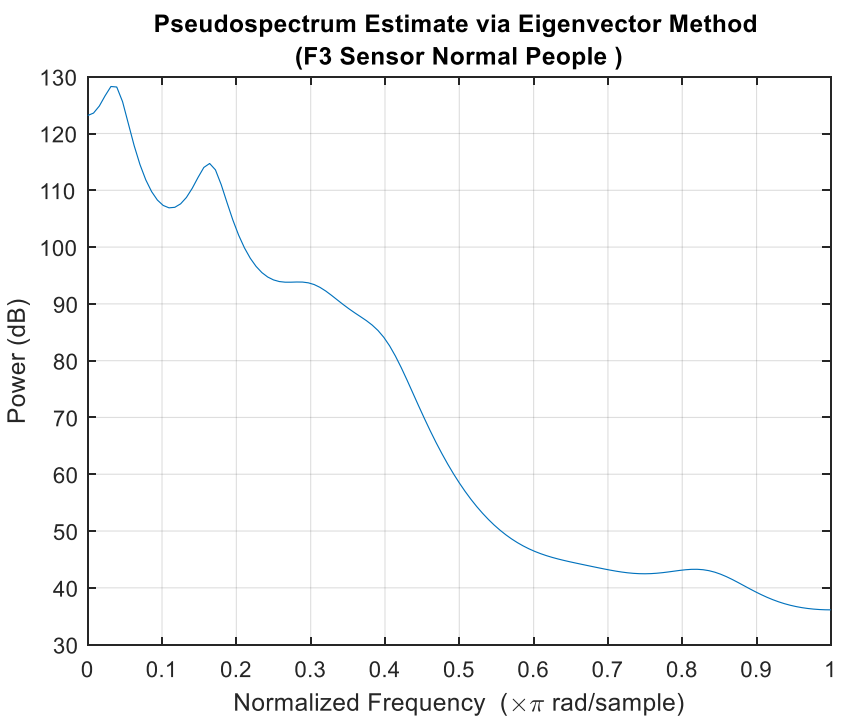

Figure 6. Pseudo-spectrum analysis for normal participants

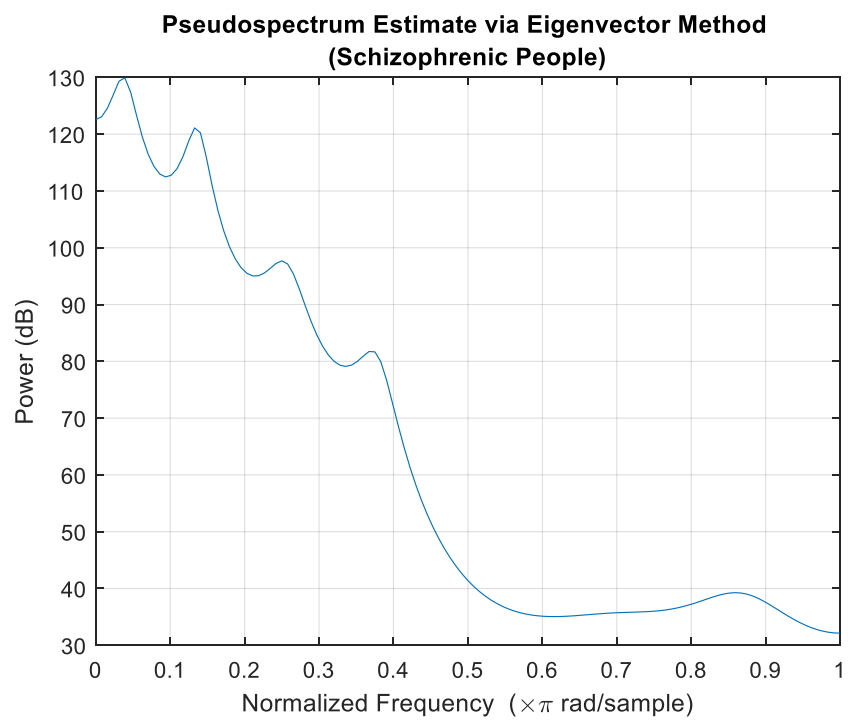

Figure 7. Pseudo-spectrum analysis for participants with schizophrenia

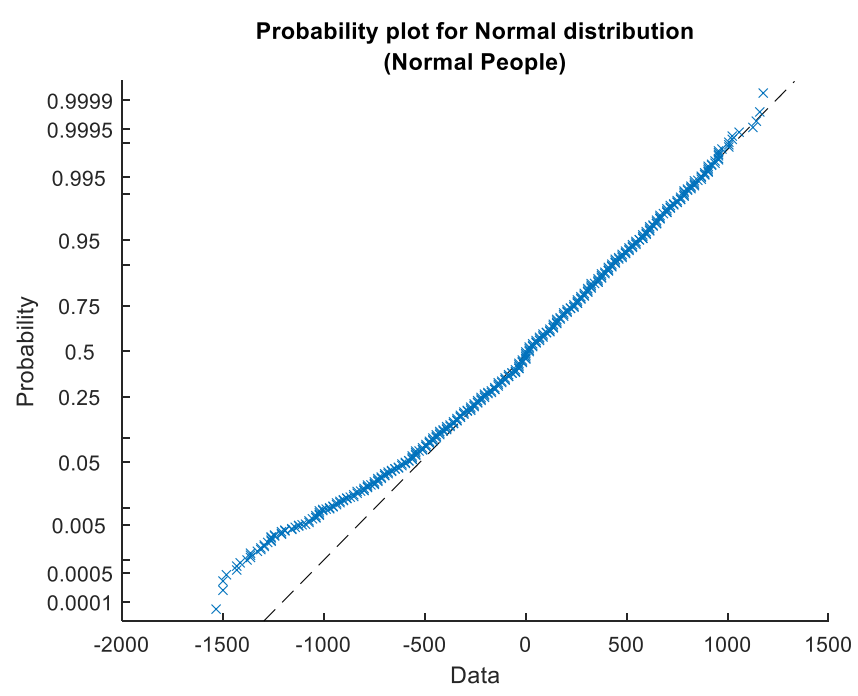

Figure 8. Probability-normal distribution for normal participants

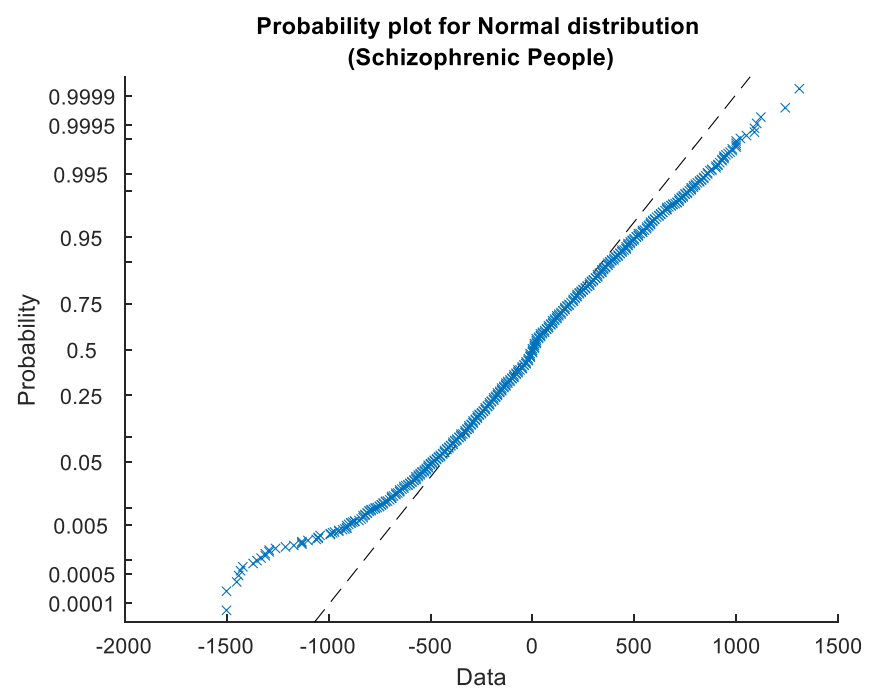

Figure 9. Probability-normal distribution for participants with schizophrenia

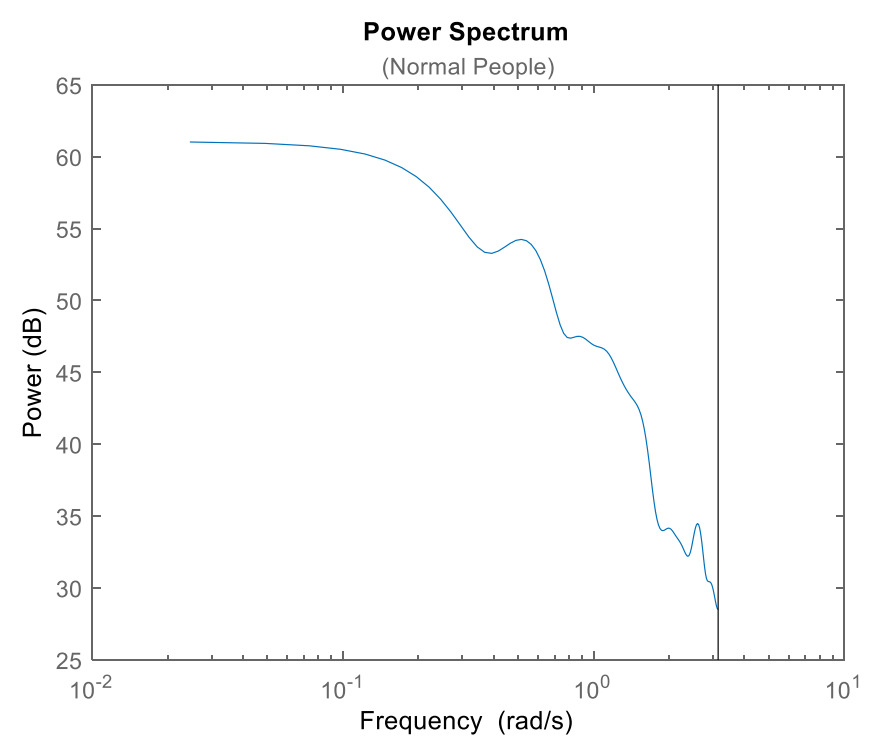

Figure 10. Power spectrum for regular participants

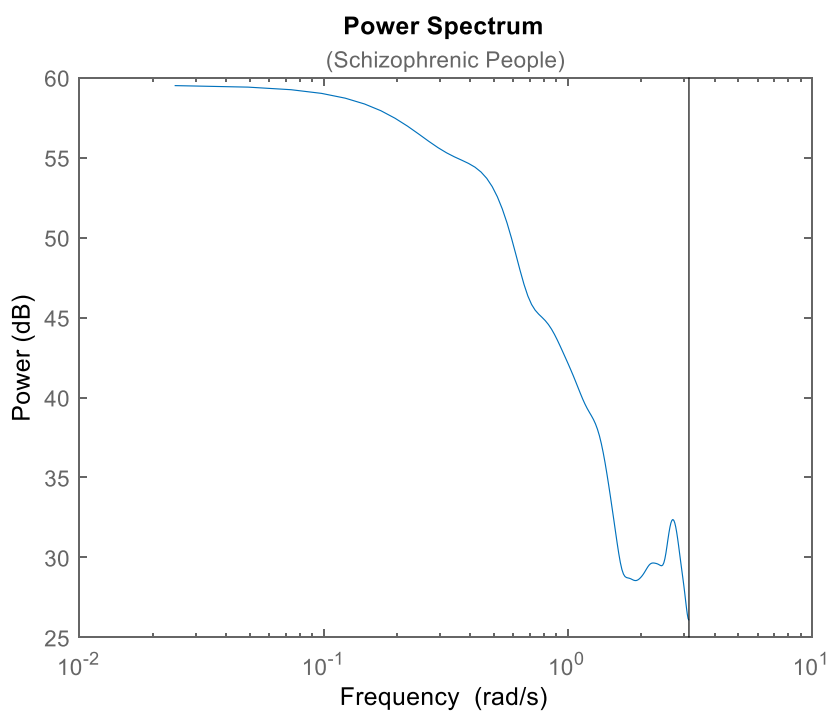

Figure 11. Power spectrum for schizophrenic participants 


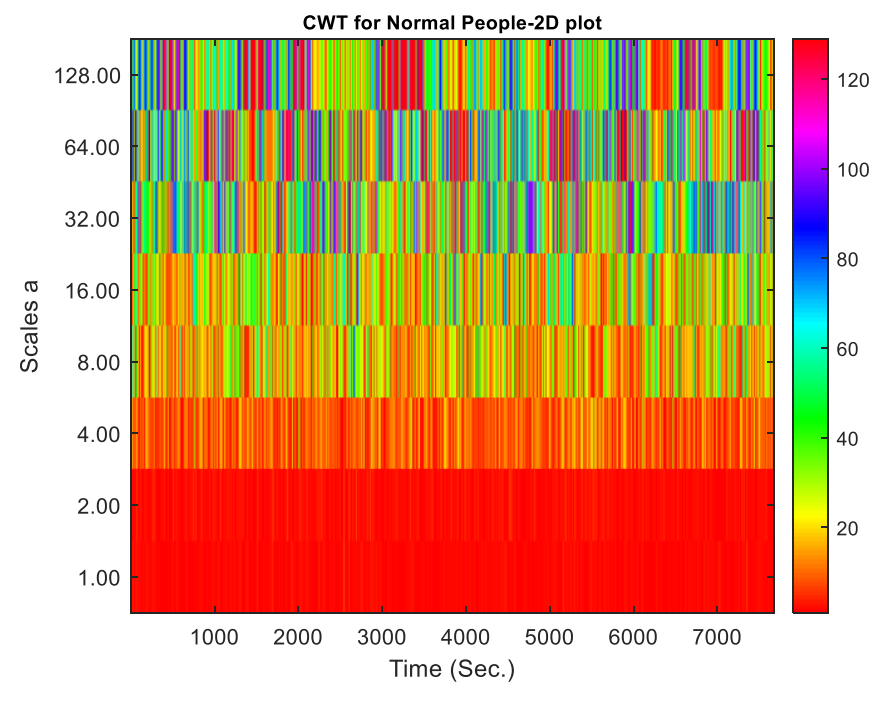

Figure 12. Continuous wavelet analysis for normal participants

The results of the Continuous Wavelet Transform (CWT) analysis are given in Figure 12 and Figure 13. In Figure 12, the analysis results for the control group (Normal) individuals, and in Figure 13, the cwt analysis of the individual diagnosed with schizophrenia is seen in detail in terms of time-scale and frequency.

Here, the regions marked on the graphic in Figure 13 can be considered as a very distinctive result for individuals diagnosed with schizophrenia. Fields corresponding to 16 and 64 values as a scale distinguish schizophrenic patients from normal control group individuals.

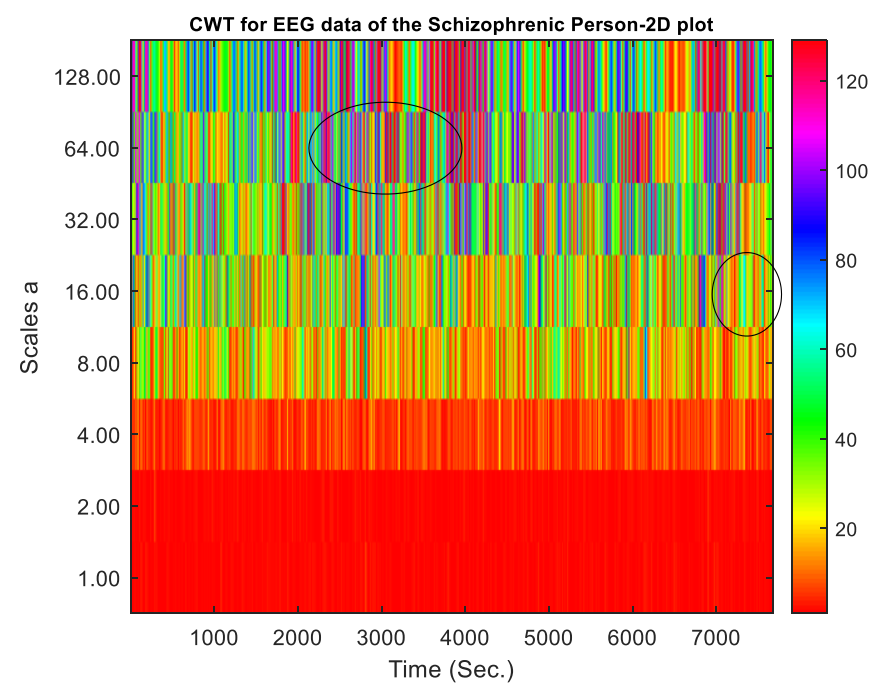

Figure 13. Continuous wavelet analysis for participants with schizophrenia

\section{DISCUSSION}

This study (http://brain.bio.msu.ru/eeg schizophrenia.htm) was conducted on the data obtained from the data bank. Motor activities of schizophrenic patients were determined in a study previously conducted by Hauge et al. [29].

Data on schizophrenic patients with deep learning were also classified by Sun et al. [30]. Spectral-based CNN analyzes of schizophrenic patients were also performed in the study conducted by Sing et al. [31]. In the study by Rösche and Fell [32] depression and schizophrenia patients were examined on P300 amplitudes. Fenton et al. Conducted one of the first studies in this context, and they extracted the spectral characteristics of the patients in their study [33]. When the studies in the literature are examined, it can be said that the studies on schizophrenia and depression have focused on the medical level or on the use of artificial intelligence [34-38]. Differently, in this study, statistical, power spectrum, normal probability and CWT analyzes of EEG data of healthy and schizophrenic patients were compared.

\section{CONCLUSIONS}

This study was conducted with EEG data from adolescents aged 10-14 years obtained from the database. Of the 84 individuals, 39 were in control and 45 were diagnosed with schizophrenia. With their eyes closed, EEG data were collected and a data bank was created and opened to public use. This study focuses on analyzing the data in this database and distinguishing between healthy and schizophrenic patients.

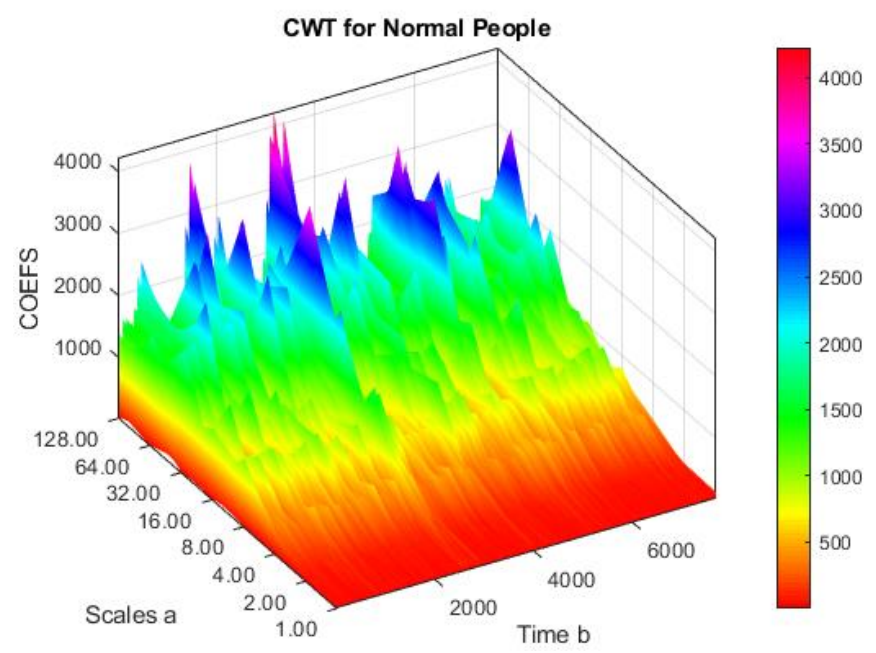

Figure 14. 3-D continuous wavelet analysis for normal participants

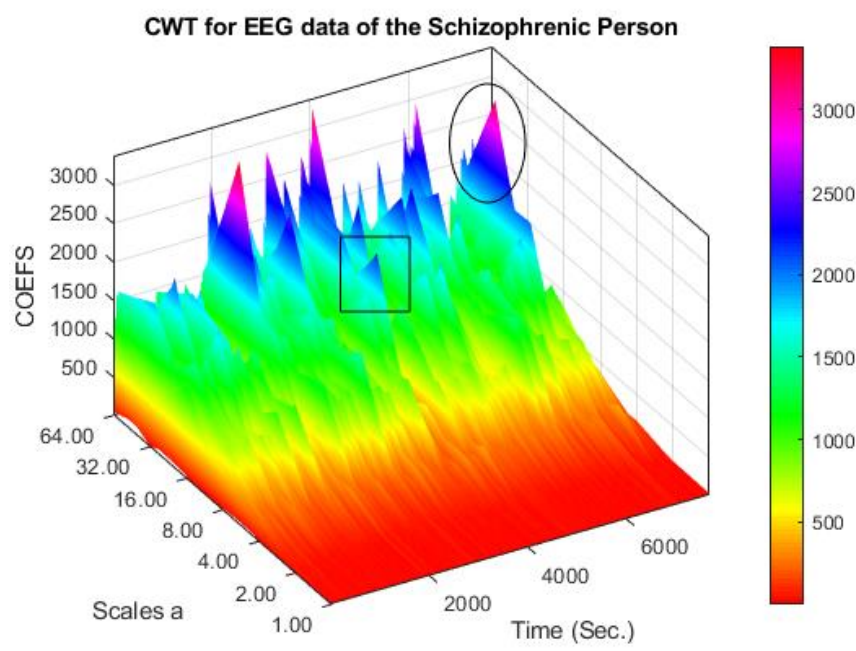

Figure 15. 3D-continuous wavelet analysis for participants with schizophrenia 
In the study, firstly, healthy and schizophrenic individuals were compared with the results of statistical analysis, and then Probability-Normal Distribution analysis and CWT analysis were performed. All of the mean, standard deviation, variance, kurtosis and skewness data made in the statistical results gave very significant results.

CWT analysis results include significant distinguishing features as shown in three dimensions in Figure 14 and Figure 15. As shown in Figure 15 in the time-scale and coefficient graph of the individual diagnosed with schizophrenia, quite successful results have been obtained in distinguishing between healthy and schizophrenic patients. However, the results of the study only deal with adolescent individuals. In this sense, these results can be recommended for diagnosis by physicians as they contain features that can be used in the diagnosis of schizophrenia patients.

\section{REFERENCES}

[1] Schmidt, C.W. (2007). Environmental connections: A deeper look into mental illness. Environmental Health Perspectives, 115(8): A404-A410. https://doi.org/10.1289/ehp.115-a404

[2] Van Winkel, R., Esquivel, G., Kenis, G., Wichers, M., Collip, D., Peerbooms, O., Rutten, B., Myin-Gereys, I., Van Os, J. (2010). Genome - wide findings in schizophrenia and the role of gene-environment interplay. CNS Neuroscience \& Therapeutics, 16(5): e185-e192. https://doi.org/10.1111/j.1755-5949.2010.00155.x

[3] Bertelsen, A., Gottesman, I.I. (1995). Schizoaffective psychoses: Genetical clues to classification. American Journal of Medical Genetics, 60(1): 7-11. https://doi.org/10.1002/ajmg.1320600103

[4] Kuperberg, G.R. (2010). Language in schizophrenia part 1: An introduction. Language and Linguistics Compass, 4(8): 576-589. https://doi.org/10.1111/j.1749818X.2010.00216.X

[5] Allen, H.A. (1983). Do positive symptom and negative symptom subtypes of schizophrenia show qualitative differences in language production? Psychological Medicine, 13(4): 787-797. https://doi.org/10.1017/s0033291700051497

[6] Ogundele M.O. (2018). Behavioural and emotional disorders in childhood: A brief overview for paediatricians. World Journal of Clinical Pediatrics, 7(1): 9-26. https://doi.org/10.5409/wjcp.v7.i1.9

[7] Fleischhacker, W.W., Arango, C., Arteel, P., Barnes, T.R., Carpenter, W., Duckworth, K., Galderisi, S., Halpern, L., Knapp, M., Marder, S.R., Moller, M., Sartorius, N., Woodruff, P. (2014). Schizophrenia-time to commit to policy change. Schizophrenia Bulletin, 40(Suppl 3): S165-S194. https://doi.org/10.1093/schbul/sbu006

[8] Dean, K., Murray, R.M. (2005). Environmental risk factors for psychosis. Dialogues in Clinical Neuroscience, $7(1)$ : 69-80. https://doi.org/10.31887/DCNS.2005.7.1/kdean

[9] Patel, K.R., Cherian, J., Gohil, K., Atkinson, D. (2014). Schizophrenia: Overview and treatment options. P \& T: A peer-reviewed Journal for Formulary Management, 39(9): 638-645.

[10] Yeisen, R.A.H., Bjornestad, J., Joa, I., Johannessen, J.O., Opjordsmoen, S. (2017). Experiences of antipsychotic use in patients with early psychosis: A two-year followup study. BMC Psychiatry, 17: 299. https://doi.org/10.1186/s12888-017-1425-9

[11] Sbargoud, F., Djeha, M., Guiatni, M., Ababou, N. (2019). WPT-ANN and belief theory based EEG/EMG data fusion for movement identification. Traitement du Signal, 36(5): 383-391. https://doi.org/10.18280/ts.360502

[12] Marzbani, H., Marateb, H.R., Mansourian, M. (2016). Neurofeedback: A comprehensive review on system design, methodology and clinical applications. Basic and Clinical Neuroscience, 7(2): 143-158. https://doi.org/10.15412/J.BCN.03070208

[13] Oh, S.L., Vicnesh, J., Ciaccio, E.J., Yuvaraj, R., Acharya UR. (2019). Deep convolutional neural network model for automated diagnosis of schizophrenia using EEG signals. Applied Sciences, 9(14): 2870. https://doi.org/10.3390/app9142870

[14] George, F.P., Shaikat, I.M., Ferdawoos, P.S., Parvez, M.Z., Uddin, J. (2019). Recognition of emotional states using EEG signals based on time-frequency analysis and SVM classifier. International Journal of Electrical \& Computer Engineering, 9(2): 2088-8708. https://doi.org/10.11591/ijece.v9i2.pp1012-1020

[15] Vasilyev, A., Liburkina, S., Yakovlev, L., Perepelkina, O., Kaplan, A. (2017). Assessing motor imagery in braincomputer interface training: Psychological and neurophysiological correlates. Neuropsychologia, 97: 56-65.

https://doi.org/10.1016/j.neuropsychologia.2017.02.005

[16] Rojas, G.M., Alvarez, C., Montoya, C.E., de la IglesiaVayá, M., Cisternas, J.E., Gálvez, M. (2018). Study of resting-state functional connectivity networks using EEG electrodes position as seed. Front Neurosci, 12: 235. https://doi.org/10.3389/fnins.2018.00235

[17] Lenssen, N. (2013). Applications of Fourier analysis to audio signal processing: An investigation of chord detection algorithms. CMC Senior Theses. Paper 704. http://scholarship.claremont.edu/cmc theses/704.

[18] Rabah, C.B., Coatrieux, G., Abdelfattah, R. (2020). Boosting up source scanner identification using wavelets and convolutional neural networks. Traitement du Signal, 37(6): 881-888. https://doi.org/10.18280/ts.370601

[19] Aslan, M.F., Sabanci, K., Durdu, A. (2016). Comparison of contourlet and time-invariant contourlet transform performance for different types of noises and images. Balkan Journal of Electrical and Computer Engineering, 7(4): 399-404. https://doi.org/10.17694/bajece.573583

[20] Majkowski, A., Kołodziej, M., Rak, R.J. (2014). Joint time-frequency and wavelet analysis-an introduction. Metrology and Measurement Systems, XXI(4): 741-758. https://doi.org/10.2478/mms-2014-0054

[21] Qian, S., Chen, D. (1999). Joint time-frequency analysis. IEEE Signal Processing Magazine, 16(2): 52-67. https://doi.org/10.1109/79.752051

[22] Dhiman R., Priyanka, Saini J.S. (2013). Wavelet analysis of electrical signals from brain: The electroencephalogram. In: Singh K., Awasthi A.K. (eds) Quality, Reliability, Security and Robustness in Heterogeneous Networks. QShine 2013. Lecture Notes of the Institute for Computer Sciences, Social Informatics and Telecommunications Engineering, vol 115. Springer, Berlin, Heidelberg. https://doi.org/10.1007/978-3-642-37949-9_24

[23] Hazarika, N., Chen, J.Z., Tsoi, A.C., Sergejew, A. (1997). 
Classification of EEG signals using the wavelet transform. Signal Processing, 59(1): 61-72. https://doi.org/10.1016/S0165-1684(97)00038-8

[24] Goswami, J.C., Chan, A.K. (1999). Fundamentals of wavelets - Theory, Algorithms and Applications. John Wiley \& Sons Inc. New York.

[25] Sadowsky, J. (1994). The continuous wavelet transform: A tool for signal investigation and understanding. Johns Hopkins APL Technical Digest, 15(4): 306-306.

[26] Komorowski, D., Pietraszek, S. (2016). The use of continuous wavelet transform based on the fast Fourier transform in the analysis of multi-channel electrogastrography recordings. Journal of Medical Systems, 40: 10. https://doi.org/10.1007/s10916-0150358-4

[27] Choi, D.A., Tagore, P., Siddiq, F., Park, K., Ewing, R. (2020). Descriptive Statistics and Visualizing Data Basic Quantitative Research Methods for Urban Planners.

[28] Warsza, Z.L., Korczyński, M.J. (2015). Statistical Properties of skewness and kurtosis of small samples from normal and two other populations. In Progress in Automation, Robotics and Measuring Techniques, pp. 293-301. Springer, Cham. https://doi.org/10.1007/978-3319-15835-8 32

[29] Hauge, E.R., Berle, J.Ø., Oedegaard, K.J., Holsten, F., Fasmer, O.B. (2011). Nonlinear analysis of motor activity shows differences between schizophrenia and depression: A study using Fourier analysis and sample entropy. PloS One, 6(1): e16291. https://doi.org/10.1371/journal.pone.0016291

[30] Sun, J., Cao, R., Zhou, M., Hussain, W., Wang, B., Xue, J., Xiang, J. (2021). A hybrid deep neural network for classification of schizophrenia using EEG data. Scientific Reports, 11(1): 1-16. https://doi.org/10.1038/s41598-021-83350-6

[31] Singh, K., Singh, S., Malhotra, J. (2021). Spectral features based convolutional neural network for accurate and prompt identification of schizophrenic patients.
Proceedings of the Institution of Mechanical Engineers, Part H: Journal of Engineering in Medicine, 235(2): 167184. https://doi.org/10.1177/0954411920966937

[32] Röschke, J., Fell, J. (1997). Spectral analysis of P300 generation in depression and schizophrenia. Neuropsychobiology, 35(2): 108-114. https://doi.org/10.1159/000119400

[33] Fenton, W.S., Hibbeln, J., Knable, M. (2000). Essential fatty acids, lipid membrane abnormalities, and the diagnosis and treatment of schizophrenia. Biological Psychiatry, 47(1): 8-21. https://doi.org/10.1016/s00063223(99)00092-x

[34] Angermeyer, M.C., Matschinger, H. (2003). Public beliefs about schizophrenia and depression: Similarities and differences. Social Psychiatry and Psychiatric Epidemiology, 38(9): 526-534. https://doi.org/10.1007/s00127-003-0676-6

[35] Berenbaum, H., Oltmanns, T.F. (1992). Emotional experience and expression in schizophrenia and depression. Journal of Abnormal Psychology, 101(1): 37-44. https://doi.org/10.1037//0021-843x.101.1.37

[36] Häfner, H., Maurer, K., Trendler, G., an der Heiden, W., Schmidt, M., Könnecke, R. (2005). Schizophrenia and depression: Challenging the paradigm of two separate diseases-A controlled study of schizophrenia, depression and healthy controls. Schizophrenia Research, 77(1): 1124. https://doi.org/10.1016/j.schres.2005.01.004

[37] Gaebel, W., Wölwer, W. (1992). Facial expression and emotional face recognition in schizophrenia and depression. European Archives of Psychiatry and Clinical Neuroscience, 242(1): 46-52. https://doi.org/10.1007/BF02190342

[38] Sinha, N., Babu, D. (2016). Statistical feature analysis for EEG baseline classification: Eyes Open vs Eyes Closed. In 2016 IEEE Region 10 Conference (TENCON), pp. 2466-2469. https://doi.org/10.1109/TENCON.2016.7848476 